\title{
Moderation of Breastfeeding Effects on Adult Depression by Estrogen Receptor Gene Polymorphism
}

\author{
Päivi Merjonen, ${ }^{1,2}$ Markus Jokela, ${ }^{1}$ Johanna Salo, ${ }^{1}$ Terho Lehtimäki, ${ }^{3,4}$ Jorma Viikari, ${ }^{5}$ \\ Olli T. Raitakari, ${ }^{6}$ Mirka Hintsanen, ${ }^{1,7}$ and Liisa Keltikangas-Järvinen ${ }^{1}$ \\ ${ }^{1}$ Unit of Personality, Work, and Health Psychology, IBS, University of Helsinki, P.O. Box 9, 00014 University of Helsinki, Finland \\ ${ }^{2}$ Department of Biological Psychology, VU University Amsterdam, 1081 BT Amsterdam, The Netherlands \\ ${ }^{3}$ Laboratory of Atherosclerosis Genetics, Department of Clinical Chemistry, Centre for Laboratory Medicine, \\ Tampere University Hospital, P.O. Box 66, 33101 Tampere, Finland \\ ${ }^{4}$ The School of Medicine, University of Tampere, 33014 Tampere, Finland \\ ${ }^{5}$ Department of Medicine, University of Turku and Turku University Hospital, 20520 Turku, Finland \\ ${ }^{6}$ Department of Clinical Physiology, Turku University Hospital and Research Centre of Applied and Preventive Cardiovascular \\ Medicine, University of Turku, P.O. Box 52, 20521 Turku, Finland \\ ${ }^{7}$ Helsinki Collegium for Advanced Studies, 00014 University of Helsinki, Finland
}

Correspondence should be addressed to Liisa Keltikangas-Järvinen, liisa.keltikangas-jarvinen@helsinki.fi

Received 4 April 2012; Accepted 2 October 2012

Academic Editor: Masha Gartstein

Copyright ( 2012 Päivi Merjonen et al. This is an open access article distributed under the Creative Commons Attribution License, which permits unrestricted use, distribution, and reproduction in any medium, provided the original work is properly cited.

\begin{abstract}
Breastfeeding is known to benefit both the mother's and the child's health. Our aim was to test the interactive effects between estrogen receptor 1 (ESR1) rs2234693 and breastfeeding when predicting the child's later depression in adulthood. A sample of 1209 boys and girls from the Young Finns Study were followed from childhood over 27 years up to age 30-45 years. Adulthood depressive symptoms were self-reported by the participants using the Beck Depression Inventory. Breastfeeding as well as several possibly confounding factors was reported by the parents in childhood or adolescence. Breastfeeding tended to predict lower adult depression, while ESR1 rs2234693 was not associated with depression. A significant interaction between breastfeeding and ESR1 was found to predict participants' depression $(P=.004)$ so that $\mathrm{C} / \mathrm{C}$ genotype carriers who had not been breastfed had higher risk of depression than T-allele carriers (40.5\% versus $13.0 \%$ ) while there were no genotypic differences among those who had been breastfed. In sex-specific analysis, this interaction was evident only among women. We conclude that child's genes and maternal behavior may interact in the development of child's adult depression so that breastfeeding may buffer the inherited depression risk possibly associated with the $\mathrm{C} / \mathrm{C}$ genotype of the ESR1 gene.
\end{abstract}

\section{Introduction}

Depression is a major mental health problem in western countries, affecting working-age young adults in particular [1]. The etiology of depression is complex and involves genetic, neurobiological, psychological, and social factors. Furthermore, recent research has demonstrated that adult vulnerability to depression may have its origins already in early childhood [2], which emphasizes the value of lifecourse studies of depression.

A large research literature has investigated the potential role of maternal behavior in the development of children's mental and behavioral problems. In particular, breastfeeding has been shown to have beneficial effects for children's physical health and cognitive development [3-5]. Emerging evidence suggests that breastfeeding might also have favorable effects on children's mental health. In a study on 5-year-olds, breastfeeding duration was associated with lower prevalence of children's mental health problems [6]. Other studies demonstrated that children who had not been breastfed in infancy had a higher risk for clinical depression in early adulthood [7] and hostility in adulthood [8].

However, genetic background may determine, in part, the sensitivity and responsiveness of individuals to environmental influences. For instance, in a study of breastfeeding 
and children's cognitive development, a polymorphism of the FADS2 gene (rs174575) was observed to moderate the beneficial effect of breastfeeding so that only individuals carrying the $\mathrm{C}$ allele of the FADS2 benefitted from breastfeeding and had higher IQ than those who had not been breastfed [9]. Genetic effects such as this may help to explain inconsistencies between different studies that have not taken genetic background into account.

In the present study, we hypothesized that a similar moderating genetic effect by estrogen-related gene might be observed in the association between breastfeeding and adult depression of offspring. We concentrated on a gene affecting estrogen functioning, estrogen receptor 1 (ESR1). Estrogen, and especially estrogen receptor functioning, has been linked to major mental health problems, for example, schizophrenia $[10,11]$ and depression $[11,12]$, and estrogen is related to breastfeeding and might therefore influence the maternal effect of breastfeeding. Estrogen receptor 1 (ESR1) gene codes for estrogen receptor $\alpha$, and the ESR1 PvuII is a functional polymorphism suggestively associated with severe mental health problems [13], for example, psychosis [10] and depression $[14,15]$. In a sample of individuals diagnosed with schizophrenia, the CC genotype of the ESR1 PvuII polymorphism was associated with lower expression of ESR1 mRNA in the prefrontal cortex [16], which may lead to an inability to respond normally to brain estrogen. However, breastfeeding and maternal care associated with it might buffer against this risk. High maternal care has been shown to increase levels of estrogen receptor $\alpha$ mRNA in female rat offspring $[17,18]$. In addition, breastfeeding might increase levels of oxytocin which has been shown to increase the expression of estrogen receptor $\alpha$ in prairie voles [19]. Thus, it is plausible that breastfeeding increases the expression of ESR1 mRNA directly or indirectly (via oxytocin) also in human brain.

The aim of this longitudinal, prospective cohort study is to test whether there is an association between breastfeeding and adult depression of offspring and whether this association is moderated by the ESR1 genotype. We hypothesized that the coincidence of environmental and genetic risk factors is most detrimental to the child's mental health, that is, that individuals who carry the possibly vulnerable CC genotype and who lack the buffering maternal effect (i.e., breastfeeding) against it, are in especially high risk to developing depression.

\section{Methods}

2.1. Participants. The present study is part of a prospective longitudinal study known as the Cardiovascular Risk in Young Finns Study, which started in 1980 with 3596 participants: boys and girls from age groups of 3, 6, 9, 12, 15 , and 18 years. Participants were derived from rural and urban surroundings in different parts of Finland to ensure geographical representativeness of the Finnish population. Complete details of the cohort have been published elsewhere $[20,21]$. There have been seven followups of the same participants, and the present study utilizes the baseline and first (in year 1983), fourth (in year 1992), fifth (in year 1997), sixth (in year 2001), and seventh (in year 2007) followups. The study was approved by local ethics committees, and all participants gave their written informed consent.

The present sample consisted of 725 women and 484 men (1209 total participants) for whom data on the ESR1 genotype, breastfeeding in childhood, and adult depression at age $30-45$ were available. Those selected for the final sample were younger (37.2 versus 37.56 years, $P=.05$ ), more often women $(60.0 \%$ versus $46.4 \% P<.001)$, had higher educated mother (10.2 versus 9.9 years, $P=.001$ ), had more often nuclear family background $(89.9 \%$ versus $83.1 \% P<.001)$, earlier birth order $(2.0$ versus $2.2 P=$ $.003)$ and fewer siblings $(2.7$ versus $2.8 P=.016)$ than those who due to missing data were left out from the analyses.

\subsection{Measures}

2.2.1. Depressive Symptoms. Depressive symptoms were selfreported by the participants in 2007 when they were $30-45$ years old using two different versions of the Beck Depression Inventory (BDI): (1) in the original 21-item BDI-II, for each item the participants choose from 4 alternative response statements the most suitable one (scored from 0 to 3 ), and the sum of these responses is calculated. Here, the BDI-II was scored both as a continuous and a dichotomous variable. The categorization of the latter was based on the previously established cut-off point for clinical depression $(0=0-13$, $1=14-63$ ) [22]. (2) In a modified version of the BDI, which has been used in several previous studies of the present cohort [23-26], participants rated 21 items of the second mildest statements of the original BDI instrument using a 5-point Likert-type scale and the mean of these responses was calculated. The modified BDI scale was also measured in 1992, 1997, and 2001, when participants were 15-30, 20-35, and 24-39 years old. Thus, the depression outcomes included (1) continuously coded BDI-II score, (2) dichotomously coded BDI-II clinical depression, and (3) continuously coded depressive symptoms of the modified BDI instrument.

2.2.2. Breastfeeding. In 1983 parents of the participants reported (1) whether the participants had been breastfed as infants $(0=$ no, $1=$ yes) and (2) the duration of breastfeeding in months. The questions of breastfeeding did not differentiate between partial and exclusive breastfeeding. Although the assessment of breastfeeding was retrospective, it has been shown that mothers' reports of breastfeeding history after 20 years are highly reliable and valid [27]. In Finland, practically all mothers and infants are regularly checked in antenatal clinics during pregnancy and in child health centers after birth from where parents get the child's personal record card with them. Thus, parents can check the information regarding breastfeeding from the participant's personal record cards obtained from child health centers. In a previous study with the present cohort, breastfed men were shown to have better brachial endothelial function than formula fed-men [28], supporting the validity of the breastfeeding measure. 
2.2.3. ESR1 rs2234693 Genotyping. Genomic DNA was extracted from peripheral blood leukocytes using a commercially available kit and Qiagen BioRobot M48 Workstation according to the manufacturer's instructions (Qiagen Inc., Hilden, Germany). Estrogen receptor 1 (ESR1, rs2234693) polymorphism was genotyped by employing the $5^{\prime}$ nuclease assay and fluorogenic allele-specific TaqMan MGB probes [29], using the ABI Prism 7900HT Sequence Detection System (Applied Biosystems, Foster City, CA, USA) and commercially available TaqMan assay (assay ID C_3163590_10). The samples were pipetted using an automated TECAN Freedom EVO-100 instrument (Tecan Group Ltd., Männedorf, Switzerland). The PCR reactions containing genomic DNA, $1 \times$ Universal PCR Master Mix, $900 \mathrm{nM}$ of each primer, and $200 \mathrm{nM}$ of each probe are performed in 384-well plates using the standard protocol in a total volume of $5 \mu \mathrm{L}$. End-point fluorescence is measured after PCR and genotype calling carried out by the allelic discrimination analysis module (ABI Prism SDS software, ABI, Foster City, CA, USA). Random duplicates were used as quality control.

2.2.4. Covariates. Mother's age at childbirth, her educational years, the family structure $(1=$ nuclear family versus $0=$ single-parent household or child of only one of the parents), number of children in family, and birth order of the child (all assessed in 1980) were included as covariates because they may have affected the mother's decision to breastfeed and indicate wider family atmosphere. Child's birth weight and whether the child was born full term $(1=$ full term $0=$ preterm) were also taken into account.

2.3. Statistical Analyses. When predicting continuously coded depression scores, the main effects of breastfeeding and ESR1, and the interaction effect between the two, were assessed with analysis of covariance, adjusted for participants' age and sex. For the dichotomous BDI-II, the corresponding analysis was carried out using logistic regression analysis. The models were then further adjusted for maternal age at childbirth, maternal educational years, family structure, number of children in family, participant's birth order, birth weight, and whether the participant was born full term. Sex differences in the ESR1-breastfeeding interaction effect were tested with three-way interactions between sex, breastfeeding, and ESR1 genotype. Given that the repeated measurements of the modified BDI were available for 4 measurement times, we also fitted a multilevel regression model in which all the measurement times were pooled together and each participant could contribute 1 to 4 person-observations to the dataset. Multilevel regression modeling takes into account the nonindependence of the observations in calculating standard errors of the estimates. All the analyses were conducted using PASW 18.0 software.

\section{Results}

Descriptive statistics for the sample are shown in Table 1. Age- and sex-adjusted partial correlations between the study variables are shown in Table 2. Having been breastfed at infancy was related to younger age of mother, being born full term, and having higher birth weight. Longer duration of breastfeeding was related to older mothers, later birth order, and higher number of children in the family.

Table 3 shows mean levels of depression by breastfeeding status and ESR1 genotype, which suggest no consistent main effects for these variables. There was a tendency for breastfeeding to be associated with a lower depression risk, particularly with depression assessed with the continuously coded BDI-II scores, but this association was not observed with other measures of depression.

Next we examined the interaction effects between ESR1 and breastfeeding in predicting depression. There was a significant interaction effect when predicting continuous BDI-II score $(F(2,1201)=5.47, P=.004)$ and a marginally significant interaction effect in the same direction when predicting dichotomous BDI-II $P=.071$ (TC: OR $(95 \%$ $\mathrm{CI})=0.74(0.18-3.01) P=.67$; CC $:$ OR $(95 \% \mathrm{CI})=5.18$ (0.92-29.14) $P=.06$; breastfed TT carriers as the reference category) and the modified BDI score $(F(2,1201)=2.28$, $P=.102)$. The age- and sex-adjusted interaction effects are illustrated in Figure 1. Among participants who had not been breastfed, individuals carrying the $\mathrm{C} / \mathrm{C}$ genotype had higher risk of BDI-II depression (40.5\% versus 13.0\%), higher BDIII mean scores (12.80 versus 5.74), and higher modified-BDI mean scores (2.55 versus 2.11 ) when compared to combined group of carriers of T/T and T/C genotypes. The $\mathrm{C} / \mathrm{C}$ genotype was not associated with increased depression risk in participants who had been breastfed. Stated the other way around, the interaction effect indicated that breastfeeding was associated with lower risk of depression in those carrying the $\mathrm{C} / \mathrm{C}$ genotype but not associated with depression in carriers of $\mathrm{T} / \mathrm{T}$ or $\mathrm{T} / \mathrm{C}$ genotypes. These interaction effects were little affected when the models were further adjusted for childhood covariates (continuous BDI-II score $(F(2,1194)=$ 5.50, $P=.004$ ), BDI-II $P=.074$ (TC:OR $(95 \%)=0.75$ $(0.18-3.09) P=.70$; CC $:$ OR $(95 \%)=5.35(0.93-30.82)$ $P=.06$; breastfed TT carriers as the reference category), and modified BDI score $(F(2,1194)=2.37, P=0.094)$.

Statistically significant three-way interaction effects between sex, breastfeeding status, and ESR1 were observed when predicting continuous BDI-II $(F(2,1196)=4.93, P=$ $.007)$, and modified BDI $(F(2,1196)=4.58, P=.010)$. However, when predicting dichotomous BDI-II the threeway interaction was not observed $(P=0.47)$. When the above analyses were fitted separately in men and women, the interaction effect was observed only in women (Figure 2). In women who had not been breastfed in infancy, carriers of the $\mathrm{C} / \mathrm{C}$ genotype had higher risk of BDI-II depression (59.8\% versus $13.9 \%)$, higher BDI-II mean scores (19.46 versus 6.37$)$, and higher modified-BDI mean scores $(3.20$ versus 2.12 ) than those carrying the $\mathrm{T} / \mathrm{T}$ or $\mathrm{T} / \mathrm{C}$ genotype.

In addition, when testing whether the interaction between ESR1 genotype and breastfeeding would replicate when predicting modified BDI in different years, replication was found when predicting BDI in $1992(F(2,1300)=4.86$, $P=.008)$ and $2001(F(2,1294)=3.28, P=.038)$. Although the interaction was not statistically significant when predicting modified BDI in $1997(F(2,1151)=1.322$, 
TABLE 1: Characteristics of the study group $(N=1209)$.

\begin{tabular}{|c|c|c|c|c|c|c|c|}
\hline \multirow{2}{*}{ Variable } & \multicolumn{2}{|c|}{ Total sample $N=1209$} & \multicolumn{2}{|c|}{ Women $N=725$} & \multicolumn{2}{|c|}{$\operatorname{Men} N=484$} & \multirow{2}{*}{$P$ value $^{\ddagger}$} \\
\hline & Value $^{\text {ta }}$ & Range & Value $^{\dagger}$ & Range & Value $^{\dagger}$ & Range & \\
\hline \multicolumn{8}{|l|}{ Family and childhood variables } \\
\hline Maternal age at childbirth & $27.30(5.57)$ & $16-48$ & $27.15(5.67)$ & $16-48$ & $27.52(5.41)$ & $18-46$ & .254 \\
\hline Maternal educational years & $10.23(3.16)$ & $2-22$ & $10.10(3.05)$ & $2-22$ & $10.43(3.31)$ & $4-22$ & .079 \\
\hline Family structure ${ }^{\S}$ & & & & & & & .315 \\
\hline Nuclear family & $1087(89.9)$ & & $657(90.6)$ & & $430(88.8)$ & & \\
\hline Other & $122(10.1)$ & & $68(9.4)$ & & $54(11.2)$ & & \\
\hline Birth order & $2.00(1.40)$ & $1-14$ & $2.01(1.42)$ & $1-11$ & $1.98(1.37)$ & $1-14$ & .663 \\
\hline Number of children in family & $2.65(1.60)$ & $1-15$ & $2.70(1.68)$ & $1-14$ & $2.59(1.47)$ & $1-15$ & .238 \\
\hline Birth status ${ }^{\S}$ & & & & & & & .599 \\
\hline Full term & $1059(87.6)$ & & $638(88.0)$ & & $421(87.0)$ & & \\
\hline Premature & $150(12.4)$ & & $87(12.0)$ & & $63(13.0)$ & & \\
\hline Birth weight & $3501(527)$ & $1040-5750$ & $3463(518)$ & $1300-5250$ & $3558(535)$ & $1040-5750$ & .002 \\
\hline Breastfeeding status ${ }^{\S}$ & & & & & & & .063 \\
\hline Breastfed & $1122(92.8)$ & & $681(93.9)$ & & $441(91.1)$ & & \\
\hline Not breastfed & $87(7.2)$ & & $44(6.1)$ & & $43(8.9)$ & & \\
\hline Duration of breastfeeding & $3.82(3.67)$ & $0-36$ & $3.99(3.87)$ & $0-36$ & $3.57(3.33)$ & $0-36$ & .048 \\
\hline \multicolumn{8}{|l|}{ Participant's adulthood variables } \\
\hline \multicolumn{8}{|l|}{$\operatorname{Sex} x^{\S}$} \\
\hline Male & $484(40.0)$ & & & & & & \\
\hline Female & $725(60.0)$ & & & & & & \\
\hline Age in 2007 & $37.21(4.91)$ & $30-45$ & $37.18(4.91)$ & $30-45$ & $37.26(4.91)$ & $30-45$ & .768 \\
\hline ESR 1 PvuII ${ }^{\S}$ & & & & & & & .052 \\
\hline $\mathrm{T} / \mathrm{T}$ & $420(34.7)$ & & $246(33.9)$ & & $174(36.0)$ & & \\
\hline $\mathrm{T} / \mathrm{C}$ & $595(49.2)$ & & 375 (51.7) & & $220(45.5)$ & & \\
\hline $\mathrm{C} / \mathrm{C}$ & $194(16.0)$ & & $104(14.3)$ & & $90(18.6)$ & & \\
\hline Depressive symptoms in 2007 & $2.06(0.66)$ & $1.00-4.67$ & $2.12(0.67)$ & $1.00-4.67$ & $1.97(0.64)$ & $1.00-4.63$ & $<0.001$ \\
\hline BDI in 2007 (continuous) & $5.24(6.41)$ & $0-46$ & $6.13(6.79)$ & $0-46$ & $3.92(5.58)$ & $0-40$ & $<0.001$ \\
\hline BDI in 2007 (dichotomous) $)^{\S}$ & & & & & & & .001 \\
\hline Depression & $127(10.5)$ & & $93(12.8)$ & & $34(7.0)$ & & \\
\hline No depression & $1082(89.5)$ & & $632(87.2)$ & & $450(93.0)$ & & \\
\hline
\end{tabular}

${ }^{\dagger}$ Values are means (and standard deviations) unless otherwise indicated.

${ }^{\ddagger}$ Gender differences, Pearson's chi-square for categorical variables, $t$-ratio for continuous variables.

$\S$ Values are numbers (and percentages) of participants.

$P=.267)$, the association was in same direction. Pooling the 4 measurement times into a single multilevel model resulted in a dataset with 5017 person-observations from 1603 unique participants. In a multilevel regression, the interaction between ESR1 genotype with breastfeeding predicted modified BDI in both only age- and sexadjusted $(F(2,1613)=3.59, P=0.028)$ and fully adjusted $(F(2,1612)=3.69, P=0.025)$ models. The three-way interaction effect between sex, breastfeeding status, and ESR1 was statistically significant $(F(2,1621)=4.721, P=0.009)$, implying that those women who had CC genotype and who had not been breastfed had higher modified-BDI mean score (2.93 versus 2.19) than T-allele carriers who had been breastfed, as suggested by the regression models for depressive symptoms in 2007 (Figure 2).

\section{Discussion}

The present study appears to be the first to show that an interaction between breastfeeding status and gene polymorphism may predict the development of child's depression later in life. The results suggest that whether or not the child is breastfed may modify the effect of estrogen receptor 1 (ESR1) rs2234693 on depression 30 to 45 years later in life. Those who were not breastfed and had the $\mathrm{C} / \mathrm{C}$ genotype had especially high risk of developing depression compared to other groups. Gender differences were also observed, the effect being evident only among women. The interaction effect was independent of a number of childhood covariates reflecting early developmental environment. 
TABLE 2: Age- and sex-adjusted correlations between variables.

\begin{tabular}{|c|c|c|c|c|c|c|c|c|c|c|c|c|}
\hline & 1 & 2 & 3 & 4 & 5 & 6 & 7 & 8 & 9 & 10 & 11 & 12 \\
\hline \multicolumn{13}{|l|}{ (1) Maternal age at childbirth } \\
\hline (2) Maternal educational years & $-.06^{*}$ & & & & & & & & & & & \\
\hline (3) Nuclear family & $.07^{*}$ & -.02 & & & & & & & & & & \\
\hline (4) Birth order & $.57 * * *$ & $-.21^{* * *}$ & .04 & & & & & & & & & \\
\hline (5) Number of children in family & $.36^{* * *}$ & $-.15^{* * *}$ & $.10^{* *}$ & $.80^{* * *}$ & & & & & & & & \\
\hline (6) Full term & .04 & -.04 & $.08^{* *}$ & $.09^{* *}$ & .05 & & & & & & & \\
\hline (7) Birth weight & $.08^{* *}$ & -.01 & $.07^{*}$ & $.11^{* * *}$ & $.06^{*}$ & $.43^{* * *}$ & & & & & & \\
\hline (8) Breastfed & $-.10^{* * *}$ & .03 & .03 & .01 & .03 & $.19^{* * *}$ & $.18^{* * *}$ & & & & & \\
\hline (9) Duration of breastfeeding & $.15^{* * *}$ & -.04 & -.00 & $.18^{* * *}$ & $.13^{* * *}$ & $.08^{* *}$ & $.08^{* *}$ & $.29 * * *$ & & & & \\
\hline (10) ESR 1 PvuII & .03 & .02 & -.01 & .02 & -.02 & .00 & $.07^{*}$ & .03 & -.01 & & & \\
\hline (11) Depressive symptoms in 2007 & -.02 & -.02 & -.03 & -.00 & .01 & .00 & -.02 & -.04 & .04 & -.03 & & \\
\hline (12) BDI in 2007 (continuous) & -.02 & .01 & -.04 & -.02 & -.03 & -.00 & -.03 & $-.06^{*}$ & .04 & .00 & $.75^{* * *}$ & \\
\hline (13) Clinical depression (BDI) & -.03 & .02 & -.03 & -.02 & -.03 & -.02 & -.03 & -.05 & .01 & -.01 & $.58^{* * *}$ & $.78^{* * *}$ \\
\hline
\end{tabular}

*** $P<0.001, * * P<0.01$, and $* P<0.05$.

TABLE 3: Age- and sex-adjusted main effects of breastfeeding and ESR1 predicting depression.

\begin{tabular}{|c|c|c|c|c|c|c|}
\hline & \multicolumn{2}{|c|}{ BDI-II dichotomous depression } & \multicolumn{2}{|c|}{ BDI-II continuous depression } & \multicolumn{2}{|c|}{ BDI modified depression } \\
\hline & OR $(95 \% \mathrm{CI})$ & $P$ & Mean (std. error) & $P$ & Mean (std. error) & $P$ \\
\hline Breastfeeding & & .054 & & .047 & & .151 \\
\hline Yes & 1.00 & & $5.14(0.19)$ & & $2.05(0.02)$ & \\
\hline No & $1.82(0.99-3.36)$ & & $6.55(0.68)$ & & $2.16(0.07)$ & \\
\hline ESR1 genotype & & .872 & & .766 & & .486 \\
\hline $\mathrm{T} / \mathrm{T}$ & 1.00 & & $5.33(0.31)$ & & $2.09(0.03)$ & \\
\hline $\mathrm{T} / \mathrm{C}$ & $0.93(0.62-1.40)$ & & $5.11(0.26)$ & & $2.04(0.03)$ & \\
\hline $\mathrm{C} / \mathrm{C}$ & $0.86(0.48-1.54)$ & & $5.45(0.46)$ & & $2.04(0.05)$ & \\
\hline
\end{tabular}

Breastfeeding may affect child's depression through at least two different mechanisms. There may be a direct nutritional link from breast milk to child's hormone and neurotransmitter functioning. Breastfeeding is known to affect child's brain development through nutritional processes involving fatty acids [30], and this effect might be mediated via hormonal processes. On the other hand, breastfeeding may be a proxy measure of maternal behavior toward the child or of a wider family atmosphere [31]. Breastfeeding mothers may be more child-oriented, have more contact with the child, and be more sensitive and less hostile toward the child. It has been suggested that mothers have higher oxytocin release after breastfeeding, and this may intensify mother's bonding to the infant [32]. Other suggestions assume that some mothers are bonded to the infant before birth, but not all [33]. A study reported that first reaction towards their newborns among about 40 percent of first time mothers was indifference, but this was changed to affection in a week among most of the mothers [34]. Thus breastfeeding may be especially important to the maternal bonding among those whose bonding did not develop during pregnancy. Early suckling and skin-toskin contact have been shown to predict better motherinfant interaction in one year of age as well as more selfregulated and less irritable children [35]. Thus, the idea of sensitive period shown in many other species may also be relevant in humans. Oxytocin is hypothesized to increase in both the mother's and the infant's brain when the infant starts to suckle the mother's nipple in skin-to-skin contact after birth [36, 37]. At least among rats, suckling alters brain functioning similarly as oxytocin administration as was observed in a study utilizing functional magnetic resonance imaging [38]. Skin-to-skin contact in form of massage has also shown to decrease aggression among preschool children [39], which might be related to oxytocin effects.

Oxytocin, on the other hand, alters estrogen expression, at least among prairie voles [19]. Lower expression of ESR1 mRNA may be related to many major mental health problems. C/C genotype of ESR1 rs2234693 is suggested to be "a risk genotype" related to schizophrenia and other psychoses $[10,16]$ and might relate to other mental health problems, like depression [14], as well. It is possible that C/C genotype carriers have inherited a vulnerability to mental health problems, which however might be neutralized by breastfeeding. Breastfeeding may increase the expression of ESR1 mRNA among C/C genotype carriers, who initially seem to have a low expression of ESR1 mRNA, and thus buffer the risk related to mental health problems among $\mathrm{C} / \mathrm{C}$ genotype carriers. Finally, as estrogen is a known female sex-hormone, the observed sex difference could be expected because estrogen might be suggested to be a more important 


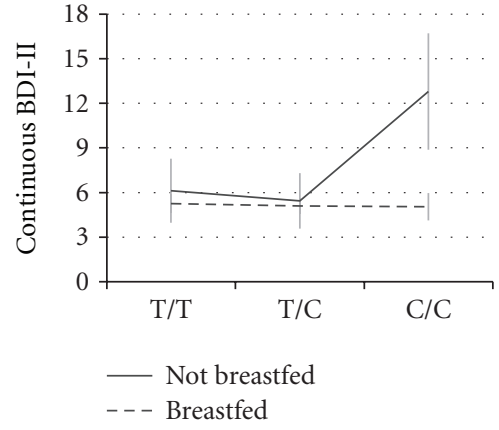

(a)

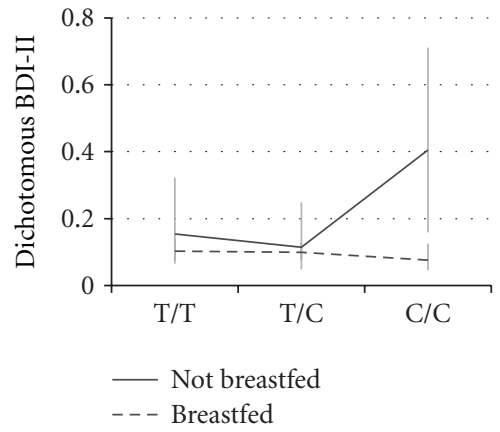

(b)

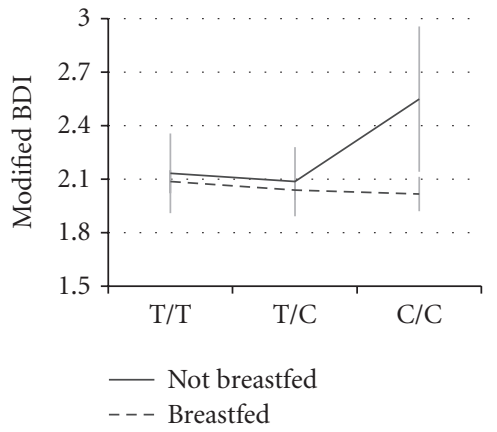

(c)

FIGURE 1: Adult depression by estrogen receptor 1 genotype (ESR1) and being breastfed at infancy. The vertical lines are $95 \%$ confidence intervals.
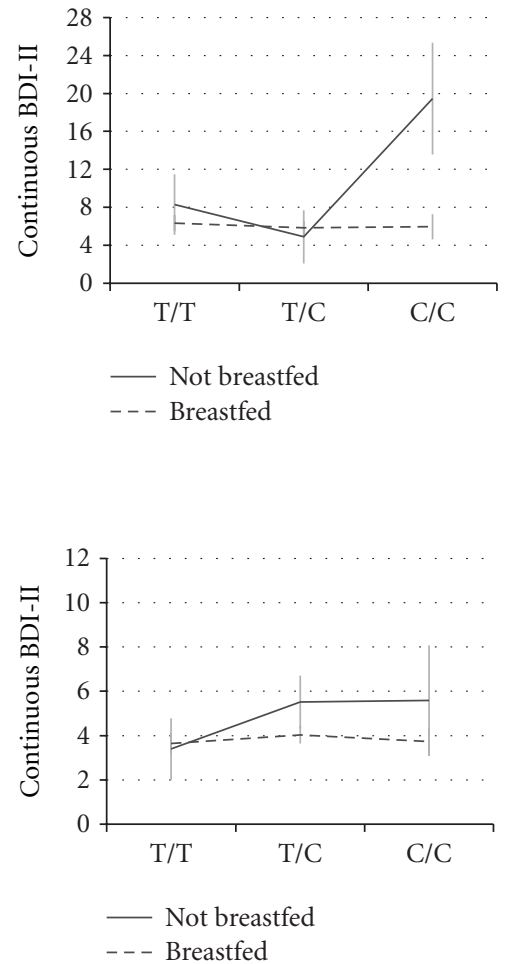

Women

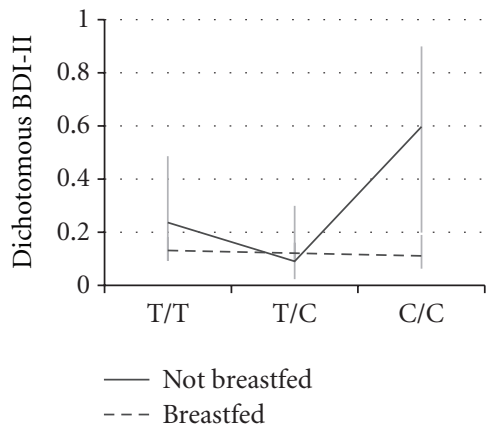

(a)

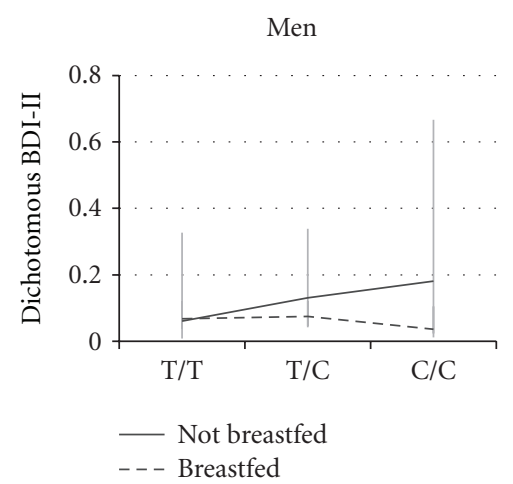

(b)
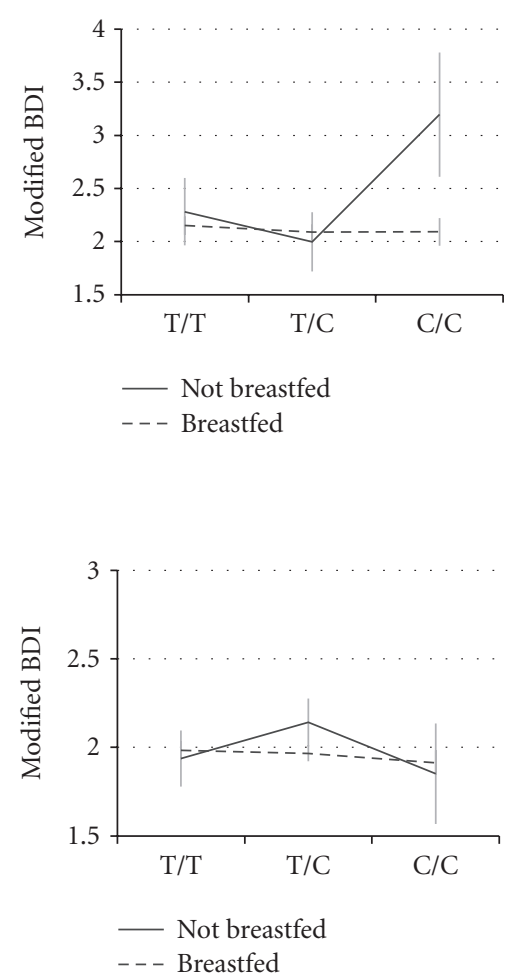

FIGURE 2: Adult depression by estrogen receptor 1 genotype (ESR1) and being breastfed at infancy separately in women and men. The vertical lines are $95 \%$ confidence intervals.

determinant of depression vulnerability in women than in men [40].

There are study limitations that need to be taken into account when interpreting the results. First, we could not control mothers' depression or their genetic background, which might introduce confounding into the results. Depressed mothers may be more reluctant to breastfeed $[41,42]$ and depression may pass from the mother to her child either via environmental factors or due to shared genetic background. Second, breastfeeding was assessed retrospectively when participants were 6-21 years old. However, maternal reports of duration of breastfeeding 20 years later have been shown to have high validity [27]. Third, due to selective dropout, those included in the final sample had a more advantageous family background, which may limit the generalizability of the results. In addition, the number of participants carrying the $\mathrm{C} / \mathrm{C}$ genotype and who were not breastfed was relatively small $(n=10)$ which increases the risk that the present results might be due to chance. Replication of these findings is therefore essential.

Our study benefits from a large population-based sample followed over a long period of time and from the use of 
two different measures of depressive symptoms. Depressive symptoms were self-reported by the participants in adulthood in 30-45 years of age while breastfeeding was reported by the participants' mothers over two decades before when participants were 6-21 years old, which reduces the influence of common informant bias to the results.

In conclusion, the present study suggests that child's genes and maternal behavior interact in the development of child's adult depression. Breastfeeding - and possibly the maternal care associated with it-may confer a protective effect especially to individuals who may have inherited a possible susceptibility to mental health problems in the form of specific estrogen receptor genotype.

\section{Abbreviations}

ESR1 gene: Estrogen receptor 1 gene

FADS2 gene: Fatty acid desaturase 2 gene

IQ: $\quad$ Intelligence quotient

mRNA: Messenger ribonucleic acid

BDI: Beck's Depression Inventory.

\section{Acknowledgments}

Ville Aalto and Irina Lisinen are acknowledged for skilful data management. This study was supported by the Academy of Finland Project 258578 (M. Hintsanen, and P. Merjonen) and 124399 (L. Keltikangas-Järvinen), Signe and Ane Gyllenberg Foundation (L. Keltikangas-Järvinen), Tampere University Hospital Medical Fund (T. Lehtimäki), Emil Aaltonen Foundation (T. Lehtimäki, M. Hintsanen), Ella \& Georg Ehrnrooth Foundation (M. Hintsanen), Research Funds of the University of Helsinki (M. Hintsanen), Research Foundation of the University of Helsinki (J. Salo), Finnish Cultural Foundation (P. Merjonen), and Alfred Kordelin Foundation (P. Merjonen).

\section{References}

[1] C. J. L. Murray and A. D. Lopez, "Alternative projections of mortality and disability by cause 1990-2020: global Burden of Disease Study," The Lancet, vol. 349, no. 9064, pp. 1498-1504, 1997.

[2] R. L. Repetti, S. E. Taylor, and T. E. Seeman, "Risky families: family social environments and the mental and physical health of offspring," Psychological Bulletin, vol. 128, no. 2, pp. 330 366, 2002.

[3] S. Ip, M. Chung, G. Raman et al., "Breastfeeding and maternal and infant health outcomes in developed countries," Evidence Report, no. 153, pp. 1-186, 2007.

[4] M. S. Kramer, F. Aboud, E. Mironova et al., "Breastfeeding and child cognitive development: new evidence from a large randomized trial," Archives of General Psychiatry, vol. 65, no. 5, pp. 578-584, 2008.

[5] E. B. Isaacs, B. R. Fischl, B. T. Quinn, W. K. Chong, D. G. Gadian, and A. Lucas, "Impact of breast milk on intelligence quotient, brain size, and white matter development," Pediatric Research, vol. 67, no. 4, pp. 357-362, 2010.

[6] M. Robinson, W. H. Oddy, J. Li et al., "Pre- and postnatal influences on preschool mental health: a large-scale cohort study," Journal of Child Psychology and Psychiatry and Allied Disciplines, vol. 49, no. 10, pp. 1118-1128, 2008.

[7] L. Batstra, J. Neeleman, C. Elsinga, and M. Hadders-Algra, "Psychiatric morbidity is related to a chain of prenatal and perinatal adversities," Early Human Development, vol. 82, no. 11, pp. 721-729, 2006.

[8] P. Merjonen, M. Jokela, L. Pulkki-Råback et al., "Breastfeeding and offspring hostility in adulthood," Psychotherapy and Psychosomatics, vol. 80, pp. 371-373, 2011.

[9] A. Caspi, B. Williams, J. Kim-Cohen et al., "Moderation of breastfeeding effects on the IQ by genetic variation in fatty acid metabolism," Proceedings of the National Academy of Sciences of the United States of America, vol. 104, no. 47, pp. 18860-18865, 2007.

[10] T. Kishi, M. Ikeda, T. Kitajima et al., "A functional polymorphism in estrogen receptor alpha gene is associated with Japanese methamphetamine induced psychosis," Progress in Neuro-Psychopharmacology and Biological Psychiatry, vol. 33, no. 5, pp. 895-898, 2009.

[11] W. R. Perlman, E. Tomaskovic-Crook, D. M. Montague et al., "Alteration in estrogen receptor $\alpha$ mRNA levels in frontal cortex and hippocampus of patients with major mental illness," Biological Psychiatry, vol. 58, no. 10, pp. 812-824, 2005.

[12] U. Halbreich and L. S. Kahn, "Role of estrogen in the aetiology and treatment of mood disorders," CNS Drugs, vol. 15, no. 10, pp. 797-817, 2001.

[13] E. E. Sundermann, P. M. Maki, and J. R. Bishop, "A review of estrogen receptor $\alpha$ gene (ESR1) polymorphisms, mood, and cognition," Menopause, vol. 17, no. 4, pp. 874-886, 2010.

[14] J. Ryan, J. Scali, I. Carrière et al., "Estrogen receptor alpha gene variants and major depressive episodes," Journal of Affective Disorders, vol. 136, pp. 1222-1226, 2012.

[15] S. J. Tsai, Y. C. Wang, C. J. Hong, and H. J. Chiu, "Association study of oestrogen receptor $\alpha$ gene polymorphism and suicidal behaviours in major depressive disorder," Psychiatric Genetics, vol. 13, no. 1, pp. 19-22, 2003.

[16] C. S. Weickert, A. L. Miranda-Angulo, J. Wong et al., "Variants in the estrogen receptor alpha gene and its mRNA contribute to risk for schizophrenia," Human Molecular Genetics, vol. 17, no. 15, pp. 2293-2309, 2008.

[17] F. A. Champagne, I. C. G. Weaver, J. Diorio, S. Sharma, and M. J. Meaney, "Natural variations in maternal care are associated with estrogen receptor $\alpha$ expression and estrogen sensitivity in the medial preoptic area," Endocrinology, vol. 144, no. 11, pp. 4720-4724, 2003.

[18] F. A. Champagne, I. C. G. Weaver, J. Diorio, S. Dymov, M. Szyf, and M. J. Meaney, "Maternal care associated with methylation of the estrogen receptor- $\alpha 1 \mathrm{~b}$ promoter and estrogen receptor$\alpha$ expression in the medial preoptic area of female offspring," Endocrinology, vol. 147, no. 6, pp. 2909-2915, 2006.

[19] K. M. Kramer, S. Yoshida, E. Papademetriou, and B. S. Cushing, "The organizational effects of oxytocin on the central expression of estrogen receptor $\alpha$ and oxytocin in adulthood," BMC Neuroscience, vol. 8, article 71, 2007.

[20] H. K. Åkerblom, M. Uhari, E. Pesonen et al., "Cardiovascular risk in young Finns," Annals of Medicine, vol. 23, no. 1, pp. 3539, 1991.

[21] O. T. Raitakari, M. Juonala, T. Rönnemaa et al., "Cohort profile: the cardiovascular risk in young Finns study," International Journal of Epidemiology, vol. 37, no. 6, pp. 1220-1226, 2008.

[22] A. T. Beck, R. A. Steer, and G. K. Brown, Depression Inventory, 2nd edition, 2009, http://cps.nova.edu/ cpphelp/BDI2.html. 
[23] M. Jokela, L. Keltikangas-Järvinen, M. Kivimäki et al., "Serotonin receptor $2 \mathrm{~A}$ gene and the influence of childhood maternal nurturance on adulthood depressive symptoms," Archives of General Psychiatry, vol. 64, no. 3, pp. 356-360, 2007.

[24] M. Jokela, T. Lehtimäki, and L. Keltikangas-Järvinen, “The influence of urban/rural residency on depressive symptoms is moderated by the serotonin receptor 2A gene," American Journal of Medical Genetics B, vol. 144, no. 7, pp. 918-922, 2007.

[25] M. Elovainio, L. Keltikangas-Järvinen, M. Kivimäki et al., "Depressive symptoms and carotid artery intima-media thickness in young adults: the cardiovascular risk in Young Finns study," Psychosomatic Medicine, vol. 67, pp. 561-567, 2005.

[26] M. Jokela, K. Räikkönen, T. Lehtimäki, R. Rontu, and L. Keltikangas-Järvinen, "Tryptophan hydroxylase 1 gene (TPH1) moderates the influence of social support on depressive symptoms in adults," Journal of Affective Disorders, vol. 100, no. 1-3, pp. 191-197, 2007.

[27] J. D. Kark, G. Troya, and Y. Friedlander, "Validity of maternal reporting of breast feeding history and the association with blood lipids in 17 year olds in Jerusalem," Journal of Epidemiology and Community Health, vol. 38, no. 3, pp. 218-225, 1984.

[28] M. J. Järvisalo, N. Hutri-Kähönen, M. Juonala et al., "Breast feeding in infancy and arterial endothelial function later in life. The cardiovascular risk in Young Finns Study," European Journal of Clinical Nutrition, vol. 63, no. 5, pp. 640-645, 2009.

[29] K. J. Livak, "Allelic discrimination using fluorogenic probes and the 5' nuclease assay," Genetic Analysis, vol. 14, no. 5-6, pp. 143-149, 1999.

[30] J. R. Marszalek and H. F. Lodish, "Docosahexaenoic acid, fatty acid-interacting proteins, and neuronal function: breastmilk and fish are good for you," Annual Review of Cell and Developmental Biology, vol. 21, pp. 633-657, 2005.

[31] S. F. Virden, "The relationship between infant feeding method and maternal role adjustment," Journal of Nurse-Midwifery, vol. 33, no. 1, pp. 31-35, 1988.

[32] J. H. Kennell and M. H. Klaus, "Bonding: recent observations that alter perinatal care," Pediatrics in Review, vol. 19, no. 1, pp. 4-12, 1998.

[33] C. M. Corter and A. S. Fleming, Psychobiology of Maternal Behavior in Human Beings, 1995.

[34] K. M. Robson and R. Kumar, "Delayed onset of maternal affection after childbirth," British Journal of Psychiatry, vol. 136, no. 4, pp. 347-353, 1980.

[35] K. Bystrova, V. Ivanova, M. Edhborg et al., "Early contact versus separation: effects on mother-infant interaction one year later," Birth, vol. 36, no. 2, pp. 97-109, 2009.

[36] M. Klaus, "Mother and infant: early emotional ties," Pediatrics, vol. 102, no. 5, pp. 1244-1246, 1998.

[37] K. Uvnäs-Moberg and M. Eriksson, "Breastfeeding: physiological, endocrine and behavioural adaptations caused by oxytocin and local neurogenic activity in the nipple and mammary gland," Acta Paediatrica, vol. 85, no. 5, pp. 525-530, 1996.

[38] M. Febo, M. Numan, and C. F. Ferris, "Functional magnetic resonance imaging shows oxytocin activates brain regions associated with mother-pup bonding during suckling," Journal of Neuroscience, vol. 25, no. 50, pp. 11637-11644, 2005.

[39] A. L. Von Knorring, A. Söderberg, L. Austin, and K. UvnäsMoberg, "Massage decreases aggression in preschool children: a long-term study," Acta Paediatrica, vol. 97, no. 9, pp. 1265$1269,2008$.
[40] J. Mill, E. Kiss, I. Baji et al., "Association study of the estrogen receptor alpha gene (ESR1) and childhood-onset mood disorders," American Journal of Medical Genetics B, vol. 147, no. 7, pp. 1323-1326, 2008.

[41] O. G. Falceto, E. R. J. Giugliani, and C. L. C. Fernandes, "Influence of parental mental health on early termination of breast-feeding: a case-control study," Journal of the American Board of Family Practice, vol. 17, no. 3, pp. 173-183, 2004.

[42] T. G. Fairlie, M. W. Gillman, and J. Rich-Edwards, "High pregnancy-related anxiety and prenatal depressive symptoms as predictors of intention to breastfeed and breastfeeding initiation," Journal of Women's Health, vol. 18, no. 7, pp. 945953, 2009. 


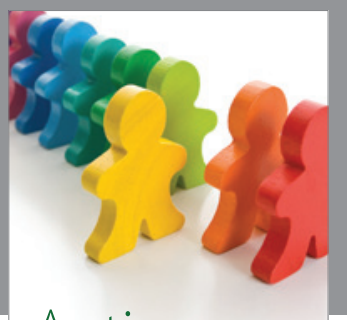

Autism

Research and Treatment
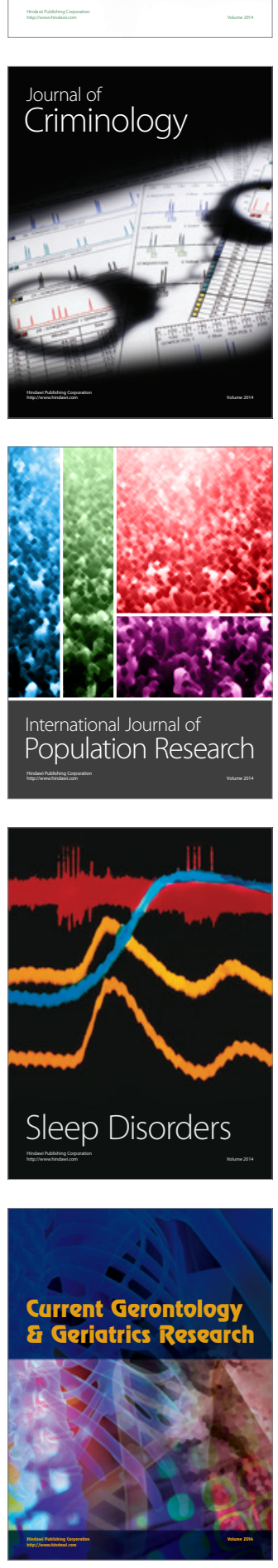
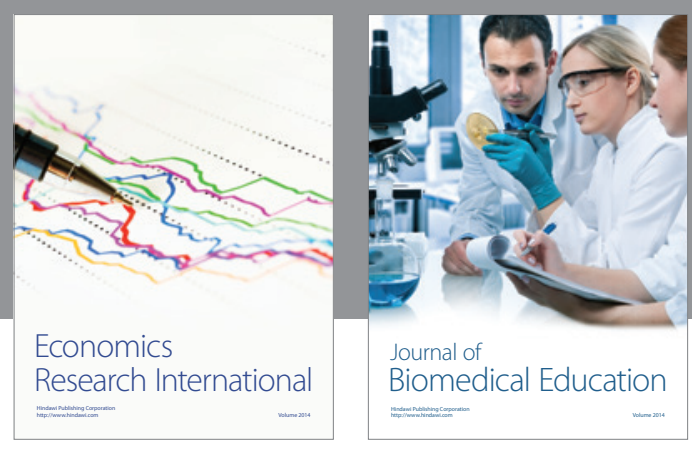

Journal of

Biomedical Education

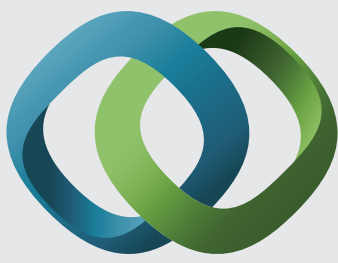

\section{Hindawi}

Submit your manuscripts at

http://www.hindawi.com
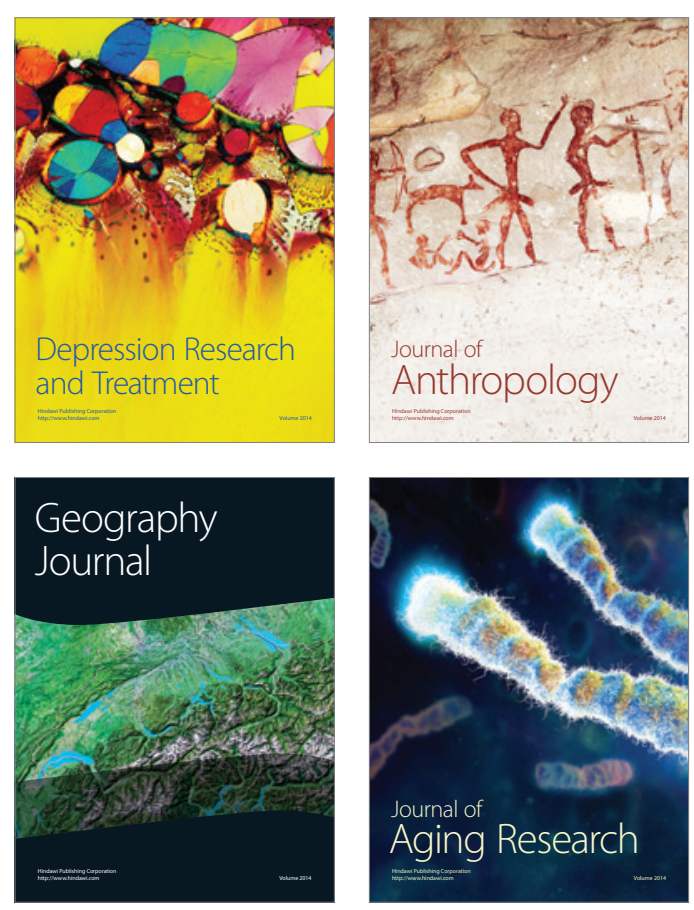

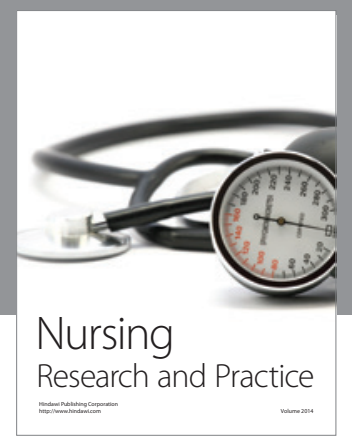

Nursing

Research and Practice

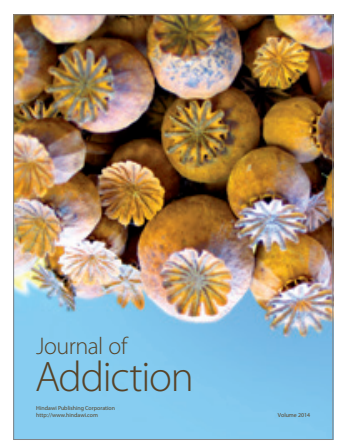

Child Development

Research

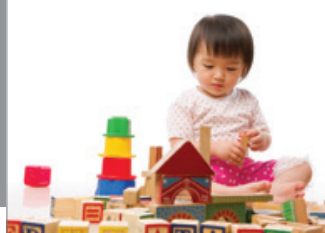

迥
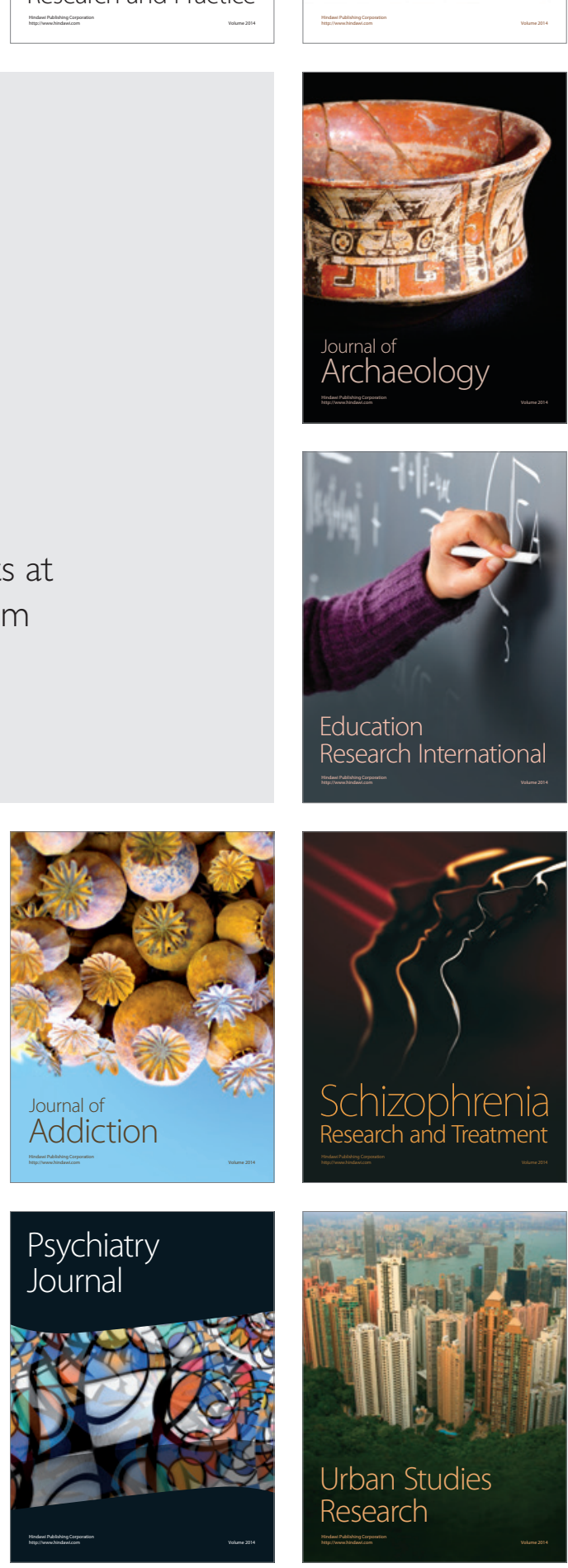